This is the accepted manuscript of the paper that is published in Current Opinion in Virology https://doi.org/10.1016/j.coviro.2020.04.006 and is available under a CC BY-NC-ND 4.0 international license.

\title{
Roles of small RNAs in the establishment of tolerant interaction between plants and viruses
}

\author{
Maja Križnik, Špela Baebler, Kristina Gruden \\ National Institute of Biology, Večna pot 111, 1000 Ljubljana, Slovenia \\ Corresponding author: kristina.gruden@nib.si
}

\begin{abstract}
In a tolerant plant-virus interaction, viral multiplication is sustained without substantial effects on plant growth or reproduction. Such interactions are, in natural environments, frequent and sometimes even beneficial for both interactors. Here we compiled evidence showing that small RNAs modulate plant immune responses and growth, hence adjusting its physiology to enable a tolerant interaction. Importantly, the role of small RNAs in tolerant interactions resembles that required for establishment of a mutualistic symbiosis. Tolerance can become a sustainable strategy for breeding for virus resistance as selection pressure for emergence of more aggressive strains is low. Understanding the processes underlying establishment of tolerance is therefore important for the development of future crops.
\end{abstract}

\section{Introduction: Tolerance in-between disease and resistance}

Plant interactions with viruses rely on interplay of different processes; processes supporting viral amplification, plant defence responses and viral counter-defence mechanisms. The balance between these defines the outcome of the interaction, resulting in either resistance (no viral multiplication) or disease (virus spreads within the plant causing dysfunction of plant metabolism). Studies of virus-plant interactions in natural environments, however, showed that plants and viruses often coexist [1]. Although a significant virus load is supported, plant growth, yield and reproduction are only minimally affected and visible symptoms are either absent or mild. This type of interaction between plant and virus is termed tolerance. Tolerance can be explained as reaching an equilibrium between different defensive and 
pathogenic processes to allow mutually advantageous compromises in host and virus fitness for long-term coexistence [2].

Plant defence against virus infections is multilayered and includes RNA silencing, pattern-triggered (PTI)- and effector-triggered (ETI)-immunity. Following virus entry into the plant cell, certain virusderived molecules are perceived as microbe-associated molecular patterns (MAMPs) by plant pattern recognition receptors (PRRs) and induce PTI [3-5]. Some viruses encode specialized effector proteins that are able to suppress this defence layer to promote their virulence [6]. In turn, many plants have evolved resistance $(R)$ genes which usually code for nucleotide-binding site-leucine-rich repeat (NBS-LRR) receptor proteins that mediate intracellular specific recognition of viral effector proteins and initiate ETI [6,7]. RNA silencing is regarded as an adaptive form of antiviral immunity [8]. Inducers of RNA silencing are virus-derived dsRNAs, which are recognized by DICER-like (DCL) proteins and then processed into virus-derived small interfering siRNAs (vsiRNAs). vsiRNAs are recruited into different Argonaute (AGO) protein(s) forming RNA-induced silencing complex (RISC) that act against viral RNA molecules. Cleaved viral RNA further serves as a template for host RNA-dependent RNA polymerase (RDR) to synthesize de novo dsRNAs thus boosting antiviral silencing effect [9]. Many viruses however adaptively evolved to evade this type of immunity by encoding viral suppressors of RNA silencing (VSRs) as viral effectors that interfere with nearly every step of the RNA silencing pathway [10-12].

RNA silencing also has an important function in regulation of plant endogenous processes (reviewed in [11]. Plants synthesize different small RNAs (sRNAs), classified into microRNAs (miRNAs) and small interfering RNAs (siRNAs) on the basis of their biogenesis and structures of their precursors. Both miRNAs and siRNAs bind to AGOs to mediate post-transcriptional gene silencing (PTGS) via sRNAdirected mRNA cleavage or translational repression, or transcriptional gene silencing (TGS) via sRNAdirected DNA methylation $[13,14]$. To date, a plethora of plant sRNAs was found to be involved in PTI and ETI [15], showing that RNA silencing, PTI and ETI are closely linked. Components of PTI/ETI can transcriptionally regulate activity of sRNAs and sRNAs regulate activity of PTI/ETI components posttranscriptionally. Functional characterization studies of sRNA's role in plant immune responses were mainly performed in plant-bacteria or plant-fungi pathosystems [16], while knowledge of the relationships between sRNAs and immune responses against viruses is still scarce. There is a vast number of reports describing how viral infection perturbs the endogenous sRNA levels, but many of these, so-called virusresponsive sRNAs, are yet to be functionally characterized [14].

While molecular mechanisms in resistance against viruses were intensively studied [17-19], the molecular basis of tolerance is much less understood. Disease can be the consequence of ineffective runaway immune response, metabolism shifts and cell rearrangements caused by viral multiplication or viral toxic 
effects (reviewed in [20]). Thus modifying any of these can result in tolerance. On the other hand, tolerance can also be a result of active recognition of the virus by receptor kinases, tuning the responses towards the favourable outcome for both organisms.

In this review, we will succinctly summarize current knowledge of sRNAs' functions governing tolerance. We also present the available knowledge on the roles of sRNAs in disease recovery, a tolerant state plants may acquire at later stages of certain plant-virus interactions. We additionally compare the involvement of sRNAs in establishment of tolerance and mutualistic symbiosis, as we argue that tolerance can be regarded as an intermediate state in symbiotic the continuum between antagonistic and mutualistic relationships.

\section{sRNAs at a crossroad: disease or tolerance}

Interestingly, many sRNAs found to date associated with the tolerance are closely interconnected either ETI or PTI immune signalling. Alternatively, tolerance promoting sRNAs are balancing trade-offs between growth and immunity.

In rice, miR444 promotes tolerance to rice stripe virus (RSV) infection. miR444 reduces accumulation of its targets, transcripts encoding MIKCC-type MADS box proteins, MADS23, MADS27, and MADS5, which are transcriptional repressors of the RDR1 gene (Figure 1) thus boosting RNA-silencing against the virus. Over-expression of miR444 resulted in milder symptoms and reduced accumulation of RSV [21]. Another monocot-specific miRNA, miR528, is involved in tolerant interaction of rice to RSV. In contrast to miR444, miR528 seems to act as a negative regulator by cleaving $L$-ascorbate oxidase $(A O)$ mRNAs, thereby reducing AO-mediated accumulation of reactive oxygen species (ROS). ROS is an important signalling component in antiviral immunity [22]. mir528 mutant plants displayed milder symptoms and accumulated less virus, whereas miR528 overexpression lines were more susceptible to RSV infection [23]. Similarly, in interaction with rice black-streaked dwarf virus (RBSDV), increased accumulation of miR528 contributed to much more severe disease symptoms, higher disease incidence and increased levels of viral RNA, whereas miR528 deficiency enhanced antiviral defence against RBSDV infection [23]. miR528 was found to be negatively regulated by the ROS, hydrogen peroxide [24], indicating that redox homeostasis is important in promoting tolerance. In diseased maize, miR528 was up-regulated after sugarcane mosaic virus infection, while miR444 was downregulated, further supporting their role as positive and negative regulators of antiviral immunity in monocots [25]. The MIR528 gene is transcriptionally activated by the SQUAMOSA Promoter-Binding-Like 9 (SPL9) transcription factor. Similarly, as the increased level of miR528, SPL9 overexpression leads to severe disease symptoms, higher disease incidence and increased virus accumulation [26]. Moreover, SPL9 is targeted by miR156, 
which when downregulated, has been linked to disease symptom occurrence in rice and maize plants in response to RSV and RBSDV (Figure 1) [27,28].

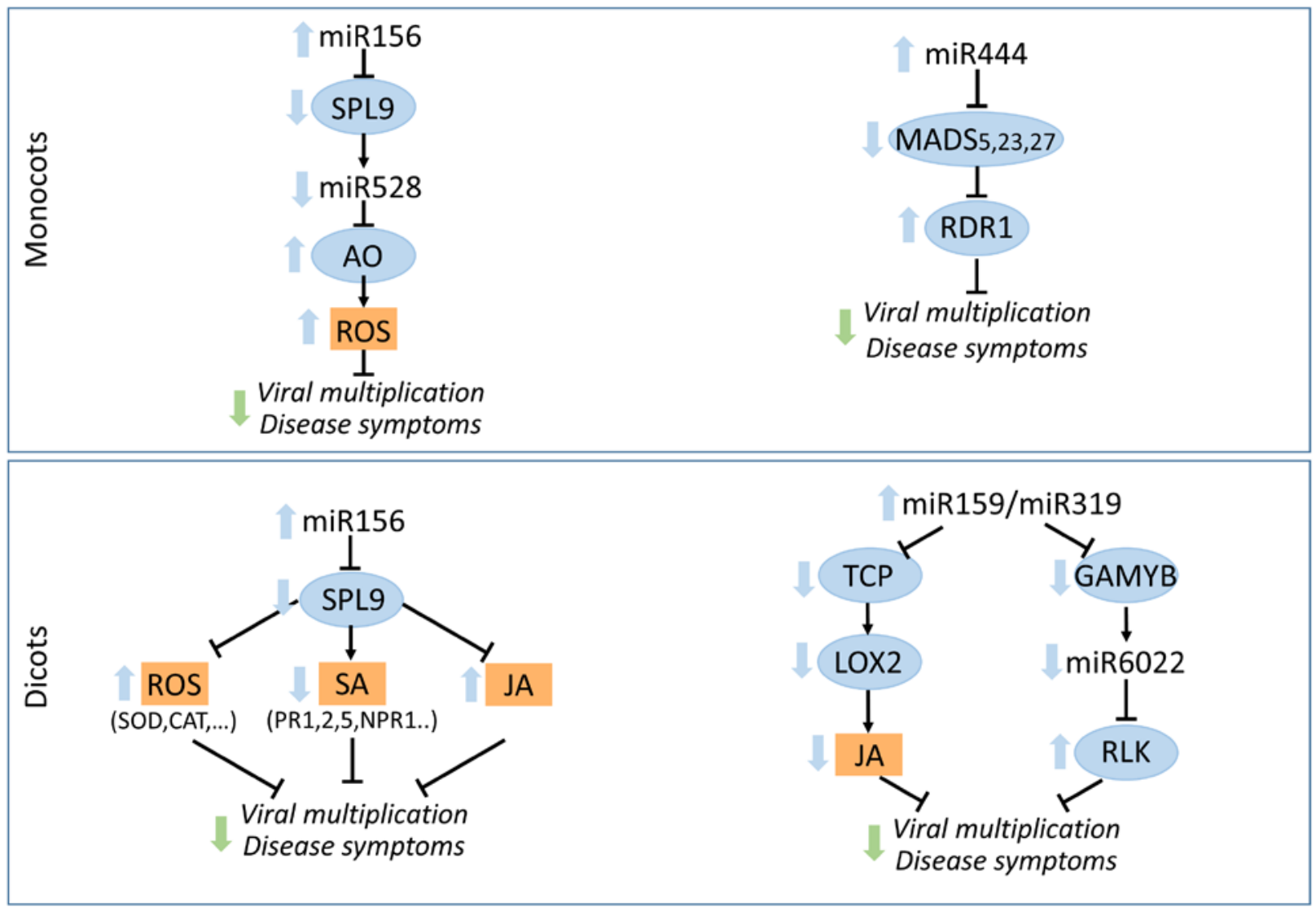

Figure 1: Role of sRNAs in plant tolerance to viral infection. Regulatory sRNA/transcript modules identified in monocots (upper panel) and in dicots (lower panel) are shown. Thick arrows indicate increase/decrease in abundance of signalling components (blue arrows) and symptoms development (green arrows), black thin arrows denote activation and bar-headed lines denote inhibition. SPL9 SQUAMOSA Promoter-Binding-Like 9 transcription factor, AO - L-ascorbate oxidase, ROS - reactive oxygen species, MADS - MIKCC-type MADS box protein, RDR - RNA-dependent RNA polymerase, SA - salicylic acid, JA - jasmonic acid, TCP - Teosinte branched1/cycloidea/proliferating bHLH transcription factor, GAMYB - MYB transcription factor controlling gibberellin signalling, LOX2 - lipoxygenase 2, RLK - receptor-like kinases.

In dicots, however, miR156 seem to function as a negative effector of immunity. Increased levels of miR156 were found to correlate with severity of disease symptoms in Nicotiana benthamiana in response to potyviruses potato virus Y (PVY) and plum pox virus, and potexvirus potato virus X [29]. Similarly, miR156 levels were significantly increased by tobacco mosaic virus, and its levels correlated with symptom severity in tobacco (Nicotiana tabacum) [30]. miR156 levels were also upregulated in tobacco plants infected with PVY [31]. Recently, miR156/SPLs regulatory module was found to control innate 
immunity by regulating ROS accumulation and activating the salicylic acid (SA) signalling pathway in Arabidopsis. mir156 suppression mutants or SPL9 overexpression mutants exhibited increased ROS levels and decreased expression of SA signalling genes [32]. When challenged with virulent Pseudomonas syringae pv. tomato DC3000strain the same plants showed less severe symptoms and lower bacterial proliferation. SA- and ROS-signalling pathways were crucial also in antiviral immunity [22,33]. Since SPL9 also negatively regulates jasmonic acid (JA) response [34] the miR156/SPL9 module must be important in controlling tolerance responses by regulating immune signalling networks through multiple connections in dicots. Even though the module has not been yet functionally characterized in response to viral infections, the elevated levels of miR156 often detected in diseased dicot plants imply that the module might function similarly as in response to bacteria [30,31,35].

Furthermore, sRNAs link immune signalling with growth. In response to PVY infection, tolerant potato plants exhibited increased levels of miR167 and a secondary siRNA (phasiRNA931) that target transcripts encoding two gibberellin (GA) biosynthesis genes, and miR319 (closely related to Arabidopsis miR159), which targets MYB33, which encodes a GAMYB transcription factor involved in GA signal transduction [36]. In Arabidopsis, a GAMYB-targeting miR159 was reported to be involved in limiting disease symptoms in response to a severe strain of CMV. Derepression of miR159 targets MYB33 and MYB65 resulted in exacerbated disease symptoms, whereas myb33/myb65 double knockout resulted in ameliorated symptoms [37]. Similarly, a diminished level of miR159 was detected in $N$. tabacum leaves displaying disease symptoms after CMV infection [38], further confirming the positive role of miR159/miR319 in the antiviral response. Additionally, there is a link between GA signalling and $R$ gene expression, supporting the hypothesis that viral perception is actively modulated in tolerant interactions. In the potato-PVY interaction, downregulation of miR6022 (targeting $L R R-R L K s$ ) occurs, which is linked to downregulation of GA signalling as GAMYB binding sites were discovered in the MIR6022 promoter region [36]. The miR159/miR319 family also targets Teosinte branched1/cycloidea/proliferating (TCP) bHLH transcription factor which targets lipoxygenase 2 (LOX2) involved in JA biosynthesis [39] (Figure 1).

In monocots, by contrast, miR319 negatively regulates tolerance responses of rice to rice ragged stunt virus and of wheat to RBSDV. miR319 overexpression facilitates infection and symptom development, whereas blocking its activity results in milder symptoms and lower virus levels [39]. Similar to miR156 and miR319, miR166 is another conserved miRNA that displays a contrasting regulatory role in antiviral responses in monocot and dicot species. In dicots, suppression of miR166 expression attenuates symptom development [30,40,41]. On the other hand, decreased miR166 levels were detected in symptomatic virusinfected rice and maize [25,42]. 


\section{Symbiosis and tolerance: are they more similar than we think?}

Rhizobial and plant arbuscular mycorrhizal (AM) symbioses represent two of the most researched mutualistic interactions, leading to development of nitrogen-fixing nodules and mycorrhizal arbuscules, respectively [43]. Recent evidence suggests that a plethora of plant sRNAs are involved in both types of symbiosis $[44,45]$. One set of regulated sRNAs is associated with negative control of $R$ genes (i.e. NBS$L R R s$ ), which is in line with the hypothesis that balancing of plant immune responses is required to tolerate invasion and proliferation of beneficial microorganisms. Decreased disease resistance gene expression and increased levels of legume-specific miRNAs targeting them (e.g. miR5213, miR5281) were observed during AM symbiosis in Medicago truncatula [46]. Moreover, miR482, which targets NBS-LRR transcripts was reported to be induced during establishment of symbiosis between soybean and Bradyrhizobium japonicum [47]. Notably, miR482 which targets NBS-LRR transcripts was upregulated following PVY infection in the tolerant potato-PVY interaction [36]. Similarity in regulation of certain sRNAs should not be so unexpected because in both, the tolerant response to viruses and mutualistic interactions, plants need to tolerate the presence of microorganisms (Figure 2).

Many miRNAs, reported to regulate rhizobial and AM symbioses, are implicated in the direct or indirect regulation of auxin signalling genes[46,48]. This is not surprising since auxin levels are crucial for proper legume nodule and arbuscule development [49,50]. Interestingly, many miRNAs, namely miR160, miR164, miR167, miR390, miR393 were found to be similarly regulated during tolerance response of potato to PVY [36]. Also, decreased GA levels were shown to be regulated by a miRNA/phasiRNA circuit in the potato tolerance response [36] and shown to be involved in rhizobial and mycorrhizal signalling network [51-53]. This is yet another similarity between the response of plants in mutualistic symbiosis and in the tolerance response (Figure 2). Several other miRNAs that were upregulated in tolerant response to PVY in potato, such as miR169, miR171 and miR319, also regulate nodulation and AM symbiosis in different legume species [46,54]. miR171 was linked to tolerance in several viral pathosystems [30,5557]. Rice overexpressing miR171 is less susceptible to RSV and shows attenuated disease symptoms, whereas reducing miR171 accumulation leads to development of disease symptoms [57]. 


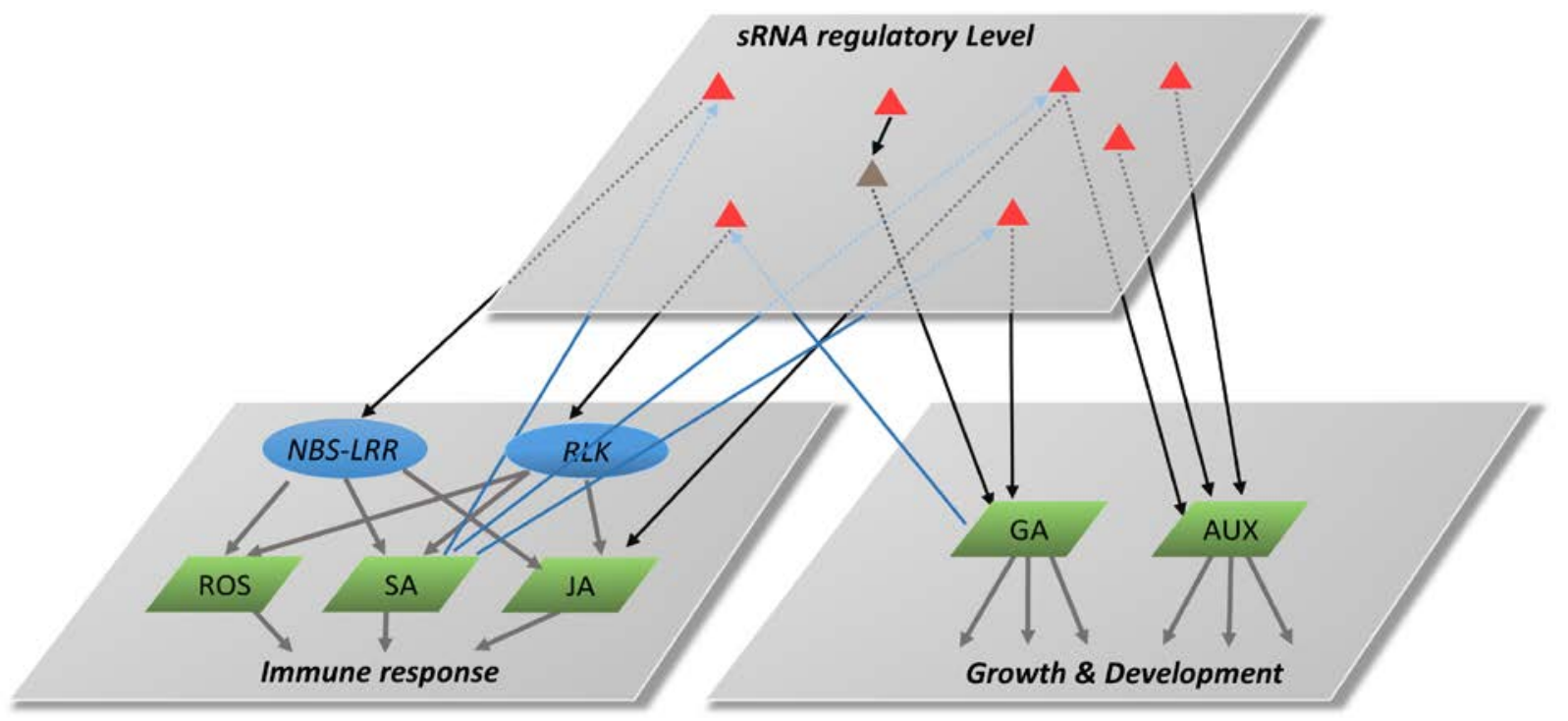

Figure 2: sRNA regulation links immunity and developmental processes in tolerance similarly as in establishment of mutualistic symbiosis. All examples of processes regulated by sRNAs in both types of interaction currently known are presented. Both, immune response and growth/developmental hormonal networks are regulated by sRNAs in both types of interaction. All regulatory levels are however tightly intertwined. miRNAs are represented by red triangles, phasiRNA is represented by a brown triangle. Arrows present relationship between sRNAs and receptors (blue ovals) or signalling modules (green rectangles), black for the regulation of transcripts by sRNA and blue for the regulation of sRNA by transcription factor in one of the modules. NBS-LRR - nucleotide-binding site-leucine-rich repeat proteins, RLK - receptor-like kinases, ROS reactive oxygen species, SA - salicylic acid, JA - jasmonic acid, - AUX - auxin, GA - gibberellin.

\section{sRNA and disease recovery: it is never too late}

Some virus-infected plants are able to recover from the disease at later stages of infection. This outcome is known as symptom recovery and is characterized by emergence of asymptomatic leaves following a systemic symptomatic infection, despite persistence of the virus [58-60]. The plant recovery phenotype thus mimics the tolerant phenotype, and disease recovery can be therefore regarded as an inducible form of tolerance [20]. One of the characteristics of recovery is that recovered leaves exhibit resistance to reinfection by a related but not unrelated viruses, suggesting that recovery is governed by a sequencespecific defence mechanism such as RNA silencing [9]. Indeed, recovery depends on AGO1 [61], the core component of antiviral silencing, on DCL4/RDR6/SGS3 PTGS pathway and on two TGS components, RDR2 and RNA polymerase IV, which are involved in the maintenance and spread of silencing [62]. Based on that, Kørner et al., proposed a model for disease recovery suggesting, that the dose of so-called 
antiviral siRNAs in tissues undergoing recovery needs to surpass a critical threshold to saturate the VSR and block its activity, ultimately allowing the recovery [62]. Antiviral siRNA are thought to primarily originate from symptomatic tissues, as they contain high amounts of viral RNA available for degradation. Accordingly, removal of symptomatic leaves was shown to delay recovery [60]. Since recovery correlated with increased vsiRNA level in recovered leaves, it was suggested that vsiRNAs are the crucial molecules causing the VSR saturation [62-64]. However, the putative involvement of plant secondary siRNAs which are also produced by DCL4/RDR6/SGS3 pathway [65] was not considered. Recent sRNA-ome analysis of PVY-infected tomato before and after symptom recovery identified many differentially regulated endogenous miRNAs and secondary siRNAs, suggesting that besides vsiRNAs, plant sRNAs seem to be important for the transition from diseased to recovered conditions as well [59]. In the case of DNA viruses, such as geminiviruses, plants additionally employ a TGS pathway [66,67]. sRNA-mediated methylation of viral genome function in restriction of the transcription and movement of the virus and also promotes a recovery process $[68,69]$.

\section{Conclusions}

For breeders, resistance is traditionally preferred over tolerance, as tolerant crops represent a virus reservoir, which might affect the sensitive varieties [20]. It was however reported that under some abiotic or biotic stress condition the latent viral infection can be even beneficial for the plant, enhancing the fitness of the host and offer protection against a larger spectrum of isolates compared to resistance [70]. Moreover, tolerance may also have an advantage over resistance for crop protection because it does not actively prevent virus infection and/or replication, therefore the selection pressure for the emergence of more aggressive strains is reduced, and it is thus likely to be more evolutionary stable than resistance [20]. sRNAs are known to modulate both immune response as well as growth and developmental signalling [36,71]. Increasing evidence shows that sRNAs govern the establishment of mutualistic symbiosis as well as tolerance, thus representing interesting markers for breeding. sRNAs, however, seem to act in complex ways, individually only contributing to a subtle effect, but by acting through multiple action points they gain their regulatory power. It seems that sRNAs are engaged with transcription factors and hormone pathways into a large network that coregulates the trade-offs between growth and immunity [72] (Figure 2). We contend that understanding this multilayered response is thus crucial for design of agriculturally efficient crops in the future. 


\section{Declarations of interest}

The authors declare no conflict of interest.

\section{Acknowledgements}

We wish to apologize to those colleagues whose work could not be cited due to space limitations. This work was supported by the Slovenian Research Agency (research core funding No. P4-0165 and projects J4-7636 and J4-1777).

\section{References}

1. Roossinck MJ, Bazán ER: Symbiosis: Viruses as Intimate Partners. Annu Rev Virol 2017, 4:123-139.

2. Bengyella L, Waikhom SD, Allie F, Rey C: Virus tolerance and recovery from viral inducedsymptoms in plants are associated with transcriptome reprograming. Plant Mol Biol 2015, 89:243-252.

3. Niehl A, Wyrsch I, Boller T, Heinlein M: Double-stranded RNAs induce a pattern-triggered immune signaling pathway in plants. New Phytol 2016, 211:1008-1019.

4. Saijo Y, Loo EP, Yasuda S: Pattern recognition receptors and signaling in plant-microbe interactions. Plant $J$ 2018, 93:592-613.

5. Niehl A, Heinlein M: Perception of double-stranded RNA in plant antiviral immunity. Mol Plant Pathol 2019, 20:1203-1210.

6. Gouveia BC, Calil IP, Machado JPB, Santos AA, Fontes EPB: Immune receptors and coreceptors in antiviral innate immunity in plants. Front Microbiol 2017, 7:1-14.

7. Dangl JL, Jones JDG: A pentangular plant inflammasome. Science 2019, 364:31-32.

8. Carr JP, Murphy AM, Tungadi T, Yoon JY: Plant defense signals: Players and pawns in plantvirus-vector interactions. Plant Sci 2018, 278:87-95.

** This work comprehensively summarises the multilayed nature of plant signaling in plant-virus-vector interactions

9. Baulcombe D: RNA silencing in plants. Nature 2004, 431:356-363.

10. Yang Z, Li Y: Dissection of RNAi-based antiviral immunity in plants. Curr Opin Virol 2018, 32:88-99.

11. Huang CY, Wang H, Hu P, Hamby R, Jin H: Small RNAs - Big Players in Plant-Microbe Interactions. Cell Host Microbe 2019, 26:173-182.

* In this review the authors compile information on endogenous function of sRNAs and RNAi machinery in plant-microbe interactions and highlight the recent discoveries of cross-kingdom RNAi

12. Gaffar FY, Koch A: Catch Me If You Can! RNA Silencing-Based Improvement of Antiviral 
Plant Immunity. Viruses 2019, 11:1-32.

* In this paper, the authors review the recent studies which reveal the enormous potential that RNAsilencing strategies hold for providing an environmentally friendly mechanism to protect crop plants from viral diseases

13. Borges F, Martienssen RA: The expanding world of small RNAs in plants. Nat Publ Gr 2015, 16:1-15.

14. Zhang B, Li W, Zhang J, Wang L, Wu J: Roles of Small RNAs in Virus-Plant Interactions. Viruses 2019, 11:1-16.

15. Fei Q, Zhang Y, Xia R, Meyers BC: Small RNAs Add Zing to the Zig-Zag-Zig Model of Plant Defenses. Mol Plant-Microbe Interact 2016, 29:165-169.

16. Song X, Li Y, Cao X, Qi Y: MicroRNAs and Their Regulatory Roles in Plant-Environment Interactions. Annu Rev Plant Biol 2019, 70:489-525.

17. de Ronde D, Butterbach P, Kormelink R: Dominant resistance against plant viruses. Front Plant Sci 2014, 5:1-17.

18. Sanfaçon H: Plant translation factors and virus resistance. Viruses 2015, 7:3392-3419.

19. Hashimoto M, Neriya Y, Yamaji Y, Namba S: Recessive resistance to plant viruses: Potential resistance genes beyond translation initiation factors. Front Microbiol 2016, 7:1-11.

20. Paudel DB, Sanfaçon H: Exploring the Diversity of Mechanisms Associated With Plant Tolerance to Virus Infection. Front Plant Sci 2018, 9:1-20.

** In this review the authors summarise processes that plants employ to allow tolerance to virus infection.

21. Wang H, Jiao X, Kong X, Hamera S, Wu Y, Chen X, Fang R, Yan Y: A signaling cascade from miR444 to RDR1 in rice antiviral RNA silencing pathway. Plant Physiol 2016, 170:2365-2377.

22. Mandadi KK, Scholthof K-BG: Plant Immune Responses Against Viruses: How Does a Virus Cause Disease? Plant Cell 2013, 25:1489-1505.

23. Wu J, Yang R, Yang Z, Yao S, Zhao S, Wang Y, Li P, Song X, Jin L, Zhou T, et al.: ROS accumulation and antiviral defence control by microRNA528 in rice. Nat Plants 2017, 3:1-7.

24. Li T, Li H, Zhang YX, Liu JY: Identification and analysis of seven H2O2-responsive miRNAs and 32 new miRNAs in the seedlings of rice (Oryza sativa L. ssp. indica). Nucleic Acids Res 2011, 39:2821-2833.

25. Xia Z, Zhao Z, Li M, Chen L, Jiao Z, Wu Y, Zhou T, Yu W, Fan Z: Identification of miRNAs and their targets in maize in response to Sugarcane mosaic virus infection. Plant Physiol Biochem 2018, 125:143-152.

26. Yao S, Yang Z, Yang R, Huang Y, Guo G, Kong X, Lan Y, Zhou T, Wang H, Wang W, et al.: Transcriptional Regulation of miR528 by OsSPL9 Orchestrates Antiviral Response in Rice. Mol Plant 2019, 12:1114-1122.

* In this paper the authors decribe a new regulatory layer of miR528-ROS signaling defence pathway.

27. Zhou Y, Xu Z, Duan C, Chen Y, Meng Q, Wu J, Hao Z, Wang Z, Li M, Yong H, et al.: Dual transcriptome analysis reveals insights into the response to Rice black-streaked dwarf virus in maize. $J$ Exp Bot 2016, 67:4593-4609. 
28. Yang J, Zhang F, Li J, Chen JP, Zhang HM: Integrative analysis of the microRNAome and transcriptome illuminates the response of susceptible rice plants to rice stripe virus. PLoS One 2016, 11:1-21.

29. Pacheco R, García-Marcos A, Barajas D, Martiáñez J, Tenllado F: PVX-potyvirus synergistic infections differentially alter microRNA accumulation in Nicotiana benthamiana. Virus Res 2012, 165:231-235.

30. Bazzini a a, Hopp HE, Beachy RN, Asurmendi S: Infection and coaccumulation of tobacco mosaic virus proteins alter microRNA levels, correlating with symptom and plant development. Proc Natl Acad Sci U S A 2007, 104:12157-12162.

31. Guo Y, Jia MA, Yang Y, Zhan L, Cheng X, Cai J, Zhang J, Yang J, Liu T, Fu Q, et al.: Integrated analysis of tobacco miRNA and mRNA expression profiles under PVY infection provids insight into tobacco-PVY interactions. Sci Rep 2017, 7:1-10.

32. Yin H, Hong G, Li L, Zhang X, Kong Y, Sun Z, Li J, Chen J, He Y: MiR156/SPL9 regulates reactive oxygen species accumulation and immune response in arabidopsis thaliana. Phytopathology 2019, 109:632-642.

33. Stare T, Ramšak Ž, Blejec A, Stare K, Turnšek N, Weckwerth W, Wienkoop S, Vodnik D, Gruden $\mathrm{K}$ : Bimodal dynamics of primary metabolism-related responses in tolerant potato-Potato virus $\mathbf{Y}$ interaction. BMC Genomics 2015, 16:1-17.

34. Mao YB, Liu YQ, Chen DY, Chen FY, Fang X, Hong GJ, Wang LJ, Wang JW, Chen XY: Jasmonate response decay and defense metabolite accumulation contributes to age-regulated dynamics of plant insect resistance. Nat Commun 2017, 8:1-13.

35. Pacheco R, García-Marcos A, Barajas D, Martiáñez J, Tenllado F: PVX-potyvirus synergistic infections differentially alter microRNA accumulation in Nicotiana benthamiana. Virus Res 2012, 165:231-235.

36. Križnik M, Petek M, Dobnik D, Ramšak Ž, Baebler Š, Pollmann S, Kreuze J, Gruden K: Salicylic Acid Perturbs sRNA-Gibberellin Regulatory Network in Immune Response of Potato to Potato virus Y Infection. 2017, 8:1-14.

37. Du Z, Chen A, Chen W, Westwood JH, Baulcombe DC, Carr JP: Using a viral vector to reveal the role of MicroRNA159 in disease symptom induction by a severe strain of Cucumber mosaic virus. Plant Physiol 2014, 164:1378-1388.

38. Lang Q, Jin C, Lai L, Feng J, Chen S, Chen J: Tobacco microRNAs prediction and their expression infected with Cucumber mosaic virus and Potato virus X. Mol Biol Rep 2011, 38:1523-1531.

39. Zhang C, Ding Z, Wu K, Yang L, Li Y, Yang Z, Shi S, Liu X, Zhao S, Yang Z, et al.: Suppression of Jasmonic Acid-Mediated Defense by Viral-Inducible MicroRNA319 Facilitates Virus Infection in Rice. Mol Plant 2016, 9:1302-1314.

40. Wang S, Cui W, Wu X, Yuan Q, Zhao J, Zheng H, Lu Y, Peng J, Lin L, Chen J, et al.: Suppression of nbe-miR166h-p5 attenuates leaf yellowing symptoms of potato virus $\mathrm{X}$ on Nicotiana benthamiana and reduces virus accumulation. Mol Plant Pathol 2018, 19:23842396.

41. Yin Z, Murawska Z, Xie F, Pawełkowicz M, Michalak K, Zhang B, Lebecka R: microRNA response in potato virus $Y$ infected tobacco shows strain-specificity depending on host and symptom severity. Virus Res 2019, 260:20-32. 
42. Du P, Wu J, Zhang J, Zhao S, Zheng H, Gao G, Wei L, Li Y: Viral infection induces expression of novel phased microRNAs from conserved cellular microRNA precursors. PLoS Pathog 2011, 7:e1002176.

43. Hussain SS, Hussain M, Irfan M, Siddique KHM: Legume, Microbiome, and Regulatory Functions of miRNAs in Systematic Regulation of Symbiosis. In Plant Microbiome: Stress Response. Edited by Egamberdieva D, Ahmad P. Springer Nature Singapore; 2018:255-282.

44. Lelandais-Briere C, Moreau J, Hartmann C, Crespi M: Noncoding RNAs , Emerging Regulators in Root Endosymbioses. 2016, 29:170-180.

45. Hobecker KV, Reynoso MA, Bustos-Sanmamed P, Wen J, Mysore KS, Crespi M, Blanco FA, Zanetti ME: The MicroRNA390/TAS3 Pathway Mediates Symbiotic Nodulation and Lateral Root Growth. Plant Physiol 2017, 174:2469-2486.

46. Devers EA, Branscheid A, May P, Krajinski F: Stars and Symbiosis : MicroRNA- and MicroRNA * -Mediated Transcript Cleavage Involved in Arbuscular Mycorrhizal Symbiosis. 2011, 156:1990-2010.

47. Li H, Deng Y, Wu TL, Subramanian S, Yu O: Misexpression of miR482, miR1512, and miR1515 increases soybean nodulation. Plant Physiol 2010, 153:1759-1770.

48. Allen E, Xie Z, Gustafson AM, Carrington JC: microRNA-Directed Phasing during TransActing siRNA Biogenesis in Plants. Cell 2005, 121:207-221.

49. Nizampatnam NR, Schreier SJ, Damodaran S, Adhikari S, Subramanian S: microRNA160 dictates stage-specific auxin and cytokinin sensitivities and directs soybean nodule development. Plant J 2015, 84:140-153.

50. Etemadi M, Gutjahr C, Couzigou JM, Zouine M, Lauressergues D, Timmers A, Audran C, Bouzayen M, Bécard G, Combier JP: Auxin perception is required for arbuscule development in arbuscular mycorrhizal symbiosis. Plant Physiol 2014, 166:281-292.

51. Floss DS, Levy JG, Lévesque-tremblay V, Pumplin N, Harrison MJ: DELLA proteins regulate arbuscule formation in arbuscular mycorrhizal symbiosis. 2013, 2013:5025-5034.

52. Fonouni-Farde C, Tan S, Baudin M, Brault M, Wen J, Mysore KS, Niebel A, Frugier F, Diet A: DELLA-mediated gibberellin signalling regulates Nod factor signalling and rhizobial infection. Nat Commun 2016, 7:1-13.

53. Jin Y, Liu H, Luo D, Yu N, Dong W, Wang C, Zhang X, Dai H, Yang J, Wang E: DELLA proteins are common components of symbiotic rhizobial and mycorrhizal signalling pathways. Nat Commun 2016, 7:1-14.

54. Lelandais-Brière C, Naya L, Sallet E, Calenge F, Frugier F, Hartmann C, Gouzy J, Crespi M: Genome-wide Medicago truncatula small RNA analysis revealed novel microRNAs and isoforms differentially regulated in roots and nodules. Plant Cell 2009, 21:2780-96.

55. Naqvi AR, Haq QMR, Mukherjee SK: MicroRNA profiling of tomato leaf curl new delhi virus (tolcndv) infected tomato leaves indicates that deregulation of mir159/319 and mir172 might be linked with leaf curl disease. Virol J 2010, 7:1-16.

56. Feng J, Wang Y, Lin R, Chen J: Altered Expression of MicroRNAs and Target mRNAs in Tomato Root and Stem Tissues upon Different Viral Infection. J Phytopathol 2013, 161:107119. 
57. Tong A, Yuan Q, Wang S, Peng J, Lu Y, Zheng H, Lin L, Chen H, Gong Y, Chen J, et al.: Altered accumulation of osa-miR171b contributes to rice stripe virus infection by regulating disease symptoms. J Exp Bot 2017, 68:4357-4367.

58. Nemes K, Gellért Á, Bóka K, Vági P, Salánki K: Symptom recovery is affected by Cucumber mosaic virus coat protein phosphorylation. Virology 2019, 536:68-77.

59. Prigigallo MI, Križnik M, Paola D De, Catalano D, Gruden K, Finetti-Sialer MM, Cillo F: Potato Virus Y Infection Alters Small RNA Metabolism and Immune Response in Tomato. Viruses 2019, 11:1-27.

* Here authors describe the role of small RNAs in establishment of recovery to virus PVY in tomato

60. Nie X, Molen TA: Host recovery and reduced virus level in the upper leaves after Potato virus $Y$ infection occur in tobacco and tomato but not in potato plants. Viruses 2015, 7:680-698.

61. Ghoshal B, Sanfaçon H: Temperature-dependent symptom recovery in Nicotiana benthamiana plants infected with tomato ringspot virus is associated with reduced translation of viral RNA2 and requires ARGONAUTE 1. Virology 2014, 456-457:188-197.

62. Kørner CJ, Pitzalis N, Peña EJ, Erhardt M, Vazquez F, Heinlein M: Crosstalk between PTGS and TGS pathways in natural antiviral immunity and disease recovery. Nat Plants 2018, 4:157-164.

*** In this study the authors demonstrate that recovery depends on the siRNA-mediated posttranscriptional gene silencing pathway and on components of a transcriptional gene silencing pathway that is known to facilitate non-cell autonomous silencing signalling. They also speculate that establishment of recovery occurs folowing robust delivery of antiviral siRNAs from source to sink tissues.

63. Rodriguez-Negrete EA, Carrillo-Tripp J, Rivera-Bustamante RF: RNA Silencing against Geminivirus: Complementary Action of Posttranscriptional Gene Silencing and Transcriptional Gene Silencing in Host Recovery. J Virol 2009, 83:1332-1340.

64. Chellappan P, Vanitharani R, Pita J, Fauquet CM: Short Interfering RNA Accumulation Correlates with Host Recovery in DNA Virus-Infected Hosts, and Gene Silencing Targets Specific Viral Sequences. J Virol 2006, 80:1064-1064.

65. Deng P, Muhammad S, Cao M, Wu L: Biogenesis and regulatory hierarchy of phased small interfering RNAs in plants. Plant Biotechnol $J$ 2018, 16:965-975.

**This review discusses the findings about biogenesis, targeting modes and regulatory networks of plant phasiRNAs

66. Ramesh S V., Sahu PP, Prasad M, Praveen S, Pappu HR: Geminiviruses and plant hosts: A closer examination of the molecular arms race. Viruses 2017, 9:1-21.

67. Raja P, Jackel JN, Li S, Heard IM, Bisaro DM: Arabidopsis Double-Stranded RNA Binding Protein DRB3 Participates in Methylation-Mediated Defense against Geminiviruses. $J$ Virol 2013, 88:2611-2622.

68. Wang C, Wang C, Zou J, Yang Y, Li Z, Zhu S: Epigenetics in the plant-virus interaction. Plant Cell Rep 2019, 38:1031-1038.

69. Coursey T, Regedanz E, Bisaro DM: Arabidopsis RNA Polymerase V Mediates Enhanced Compaction and Silencing of Geminivirus and Transposon Chromatin during Host Recovery 
from Infection. $J$ Virol 2018, 92:1-18.

70. Aguilar E, Cutrona C, del Toro FJ, Vallarino JG, Osorio S, Pérez-Bueno ML, Barón M, Chung BN, Canto T, Tenllado F: Virulence determines beneficial trade-offs in the response of virusinfected plants to drought via induction of salicylic acid. Plant Cell Environ 2017, 40:29092930.

71. Deng Y, Wang J, Tung J, Liu D, Zhou Y, He S, Du Y, Baker B, Li F: A role for small RNA in regulating innate immunity during plant growth. 2018, 14:1-22.

72. Ning Y, Liu W, Wang GL: Balancing Immunity and Yield in Crop Plants. Trends Plant Sci 2017, 22:1069-1079. 